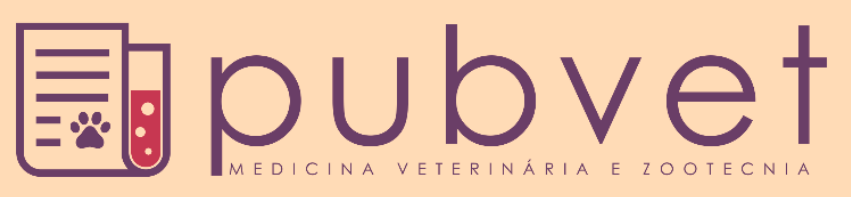

https://doi.org/10.31533/pubvet.v14n10a670.1-6

\title{
Aspectos clínicos e cirúrgicos do parasitismo por Dioctophyma renale em cão
}

Camila Mariellen Evangelista ${ }^{19}$, Chaiane Wyzykowski Soares ${ }^{2}$, Rodrigo Polido de Araújo ${ }^{2}$, Pietra Viertel Molinari ${ }^{3} \mathbb{9}$, Bruna Reinert ${ }^{4}$, Sarah Luiza Soares ${ }^{4} \sqrt{9}$, Katherinne Barth Wanis Figueiredo $^{4}$, Juliana de Abreu Pereira ${ }^{5 *}\left(\mathbb{0}\right.$, Franciolle Dias Pedras $^{60}$

${ }^{I}$ Médica Veterinária, mestre em Ciência Animal pela Universidade do Estado de Santa Catarina (UDESC), Campus Lages

${ }^{2}$ Médico Veterinário, Clínica Veterinária Bicho de Estimação, Joinville, Santa Catarina

${ }^{3}$ Discente do curso de Medicina Veterinária do Instituto Federal Catarinense (IFC) campus Araquari

${ }^{4}$ Discente do curso de Medicina Veterinária da Universidade Sociedade Educacional de Santa Catarina (UNISOCIESC) campus Joinville

${ }^{5}$ Docente do curso de Medicina Veterinária da UNISOCIESC campus Joinville.

${ }^{6}$ Médica Veterinária, atuante na área de ultrassonografia veterinária, VetScanner-SC, Joinville

*Autor para correspondência, E-mail: juliana.abreu@unisociesc.com.br

Resumo. Dioctophyma renale é o maior nematódeo que parasita o rim de mamíferos domésticos, silvestres e humanos, mas é encontrado principalmente em carnívoros piscívoros. É uma parasitose que afeta com mais frequência o rim direito, podendo causar insuficiência renal e levar o animal a morte. Dessa forma, este trabalho teve como objetivo relatar o caso clínico de um cão, sem raça definida (SRD), macho, com idade estimada de 10 anos e peso de 8 quilos do munícipio de Joinville e descrever o procedimento cirúrgico da nefrectomia realizada para a retirada do parasito. O diagnóstico da presença de Dioctophyma renale foi um achado acidental, após ser realizada uma ultrassonografia, por outras suspeitas e devido a maiores problemas de saúde os quais o cão apresentava, morreu dias após a realização da cirurgia.

Palavras-chave: dioctofimose, rim, nefrectomia

\section{Clinical and surgical aspects of Dioctophyma renale parasitism in a dog}

\begin{abstract}
Dioctophyma renale is the largest nematode that parasites the kidney of domestic, wild, and human mammals, but is found mainly in piscivorous carnivores. It is a parasite that affects more frequently the right kidney, which can cause kidney failure and lead the animal to death. The objective of this study was to report the clinical case of a male dog with 10 years of age and weight of $8 \mathrm{~kg}$ of Joinville municipality and to describe the surgical procedure of the nephrectomy performed for the removal of the parasite. The diagnosis of the presence of Dioctophyma renale was an accidental finding, after being performed ultrasonography, by other suspicions, and due to the greater health problems, that the dog presented, died days after the surgery.
\end{abstract}

Keywords: dioctophimosis, kidney, nephrectomy

\section{Aspectos clínicos y quirúrgicos del parasitismo por Dioctophyma renale en un perro}

Resumen. Dioctophyma renale es el nematodo más grande que parasita el riñón de mamíferos domésticos, salvajes y humanos, pero se encuentra principalmente en carnívoros que se alimentan de peces. Es una parasitosis que afecta con mayor frecuencia al riñón derecho, lo que puede causar insuficiencia renal y llevar al animal a la muerte. Por lo tanto, este estudio tuvo como objetivo informar el caso clínico de un perro mestizo, 
macho, con una edad estimada de 10 años y un peso de 8 kilos del municipio de Joinville y describir el procedimiento quirúrgico de la nefrectomía realizada para la eliminación del parásito. El diagnóstico de la presencia de Dioctophyma renale fue un hallazgo accidental, después de que se realizó una ecografía, debido a otras sospechas y debido a los mayores problemas de salud que tenía el perro, murió días después de la cirugía.

Palabras clave: dioctofimosis, riñón, nefrectomía

\section{Introdução}

Dioctophyma renale (D. renale), conhecido como verme gigante do rim, é o maior nematódeo já descrito, o qual parasita mamíferos domésticos, selvagens; e inclusive humanos (Katafigiotis et al., 2013). Pode chegar a medir aproximadamente 100 centímetros $(\mathrm{cm})$ de comprimento por $1,2 \mathrm{~cm}$ de diâmetro e, na maioria das vezes, é encontrado no rim direito. Este parasito possui a habilidade de destruir o parênquima renal, onde o mesmo encontra-se imerso em sangue, restando apenas a cápsula do órgão (Monteiro et al., 2002).

Além do rim, o D. renale foi observado livre na cavidade abdominal, útero, vesícula urinária e glândulas mamárias (Zardo et al., 2012). Os caninos são considerados os principais hospedeiros, principalmente os que possuem hábitos errantes e se alimentam de peixes e crustáceos aquáticos infectados com o anelídeo oligoqueta, hospedeiro intermediário do D. renale (Birchard \& Sherding, 2008; Ruiz et al., 2014).

Os sinais clínicos em animais que foram infectados são: fraqueza, apatia, emaciação, hematúria e dores lombares (Colpo et al., 2007; Lemos et al., 2010; Nakagawa et al., 2007). O diagnóstico pode ser realizado através da urinálise, para detectar ovos do parasita, e ultrassonografia abdominal, para averiguar o aspecto do rim e demais órgãos que possam estar comprometidos. O tratamento é realizado através da cirurgia, retirando o parasito do rim acometido ou até mesmo com a remoção do rim injuriado (Ruiz et al., 2014). O trabalho foi realizado com o objetivo de relatar o caso de um cão errante infestado pelo parasito $D$. renale.

\section{Relato de caso}

Um cão, sem raça definida (SRD), macho, com idade estimada de 10 anos, peso corporal de $8 \mathrm{~kg}$ foi encontrado na rua e encaminhado a uma Organização Não Governamental (ONG) da cidade de Joinville, Santa Catarina (dia 0 - D0). O animal apresentava maciça infestação por ectoparasitos (pulgas e carrapatos). No dia seguinte (D1), por apresentar inapetência e fraqueza, sem conseguir ficar de pé, foi encaminhado à uma clínica veterinária.

Durante o atendimento, o animal foi submetido a exame físico onde foi constatada desidratação grave, temperatura de $39,3{ }^{\circ} \mathrm{C}$ e mucosas pálidas; levando à suspeita de anemia. Para complementar o exame clínico realizou-se um hemograma (Tabela 1), onde foi confirmada a suspeita de anemia associada à infecção por Ehrlichia canis, sendo visualizadas mórulas nos leucócitos. Para o tratamento foi recomendada transfusão sanguínea imediata do volume de $400 \mathrm{~mL}$ de sangue total. Após o procedimento o animal demonstrou-se mais ativo, tendo interesse por alimento.

Treze dias após o primeiro atendimento (D14), o cão retornou à clínica com estado clínico mais grave; onde foram repetidos os exames de hemograma e bioquímica (Tabela 1) e feita nova transfusão, semelhante à anterior. Além disso, foi realizada contagem de reticulócitos, onde o valor encontrado foi indicativo de anemia regenerativa. Através do hemograma foi possível classificar a anemia como microcítica hipocrômica e notar uma piora na leucocitose. Para as análises de bioquímica sérica, os resultados encontrados estiveram dentro dos padrões de normalidade

Para verificar a existência de algum nódulo interno, que justificasse a piora tão rápida do quadro, foi realizado o exame de ultrassonografia, identificando no rim direito indícios de parasitismo por $D$. renale. As alterações ultrassonográficas estão representadas na Figura 1.

Após o diagnóstico, o animal foi submetido à nefrectomia. $\mathrm{O}$ animal foi posicionado em decúbito dorsal e, após a realização da antissepsia e preparo do local, foi realizada a incisão no abdome na linha mediana (pré-retroabdominal). Foi utilizado um afastador abdominal auto-estático para aumentar a área 
de exposição. $\mathrm{O}$ duodeno foi tracionado para o exterior da cavidade abdominal para facilitar a exposição do rim direito. Em seguida o retroperitônio foi penetrado entre o rim direito e a veia cava caudal. $\mathrm{O}$ hilo renal foi dissecado para expor a veia renal e o ureter, os quais foram duplamente ligados com fio de sutura sintético não absorvível 2-0 Nylon ${ }^{\circledR}$. Após secção da veia renal e do ureter, foi localizada a artéria renal, que também foi duplamente ligada e seccionada. Em seguida, foi realizada a retirada do rim direito. Após a remoção cirúrgica, foi verificada a presença do parasito no rim acometido conforme evidenciado na Figura 2.

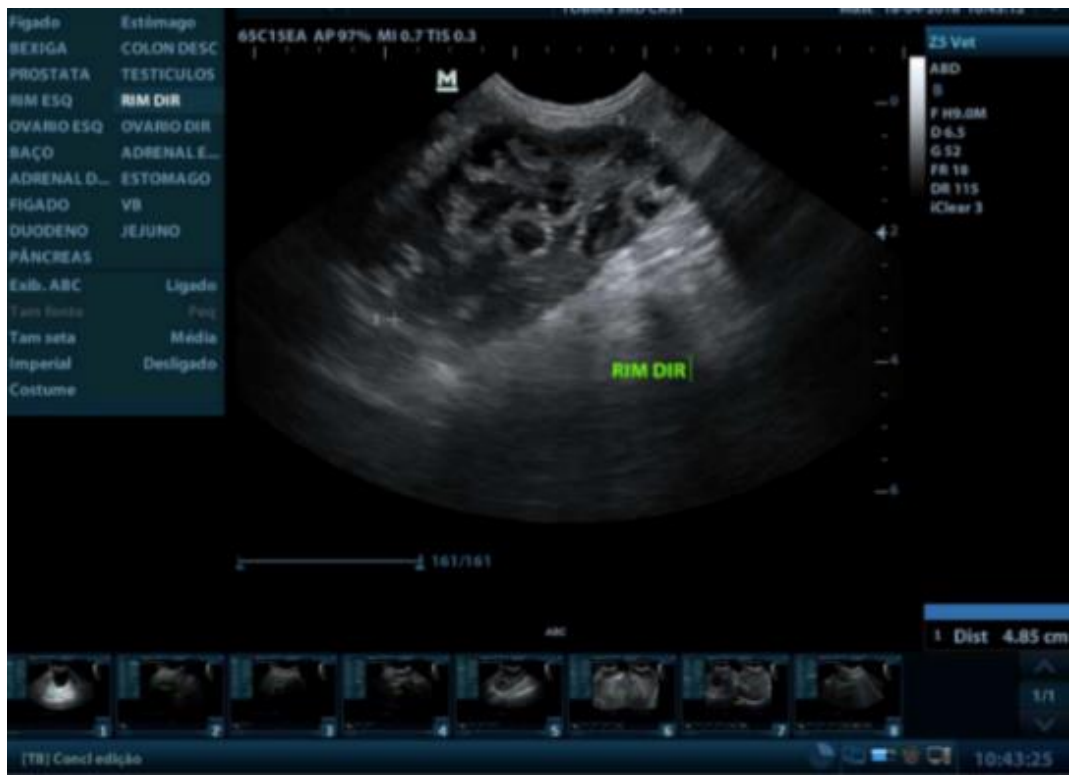

Figura 1. Aspecto ultrassonográfico de rim direito de cão errante acometido por dioctofimose. São evidenciadas perda da arquitetura interna do órgão, bem como a visualização do parasito em diversos cortes transversais.

Tabela 1. Resultados de exames laboratoriais (hemograma e bioquímica) do paciente ao dia 1 (D1) e ao dia 14 (D14) de acompanhamento. Valores de referência: Jain (1993) e Kaneko et al. (2008).

\begin{tabular}{lccc}
\hline Parâmetro & Resultado D1 & Resultado D14 & Referência \\
\hline Hematócrito (Ht) & 6 & 7 & $37-55 \%$ \\
Hemácias & 1,08 & 1,02 & $5,5-8,5{\mathrm{milhões} / \mathrm{mm}^{3}}$ \\
Hemoglobina (Hb) & 2,3 & 1,9 & $12-18 \mathrm{~g} / \mathrm{dL}$ \\
Volume corpuscular médio & 59,0 & 58,7 & $60-77 \mathrm{fL}$ \\
Concentração de hemoglobina corpuscular média & 28,7 & 20 & $32-36 \%$ \\
Leucometria global & 21.300 & 38.400 & $6,000-17,000 / \mathrm{mm}^{3}$ \\
Metamielócitos & 0 & 0 & $0 / \mathrm{mm}^{3}$ \\
Bastões & 213 & 1.152 & $0-510 / \mathrm{mm}^{3}$ \\
Neutrófilos segmentados & 16.827 & 32.640 & $3,000-11,500 / \mathrm{mm}^{3}$ \\
Linfócitos & 2.343 & 3.072 & $1,000-4,800 / \mathrm{mm}^{3}$ \\
Monócitos & 1.917 & 1.536 & $150-1,350 / \mathrm{mm}^{3}$ \\
Eosinófilos & 0 & 0 & $100-1,250 / \mathrm{mm}^{3}$ \\
Basófilos & 0 & 0 & $0-170 / \mathrm{mm}^{3}$ \\
Proteína plasmática total (PPT) & 8,0 & 7,6 & $54-71 \mathrm{~g} / \mathrm{dL}$ \\
Plaquetas & 144.000 & 268.000 & $144,000-510,000 / \mathrm{mL}^{3}$ \\
\hline Alanina aminotransferase & 228 & 129 & $0-102 \mathrm{U} / \mathrm{L}$ \\
Creatinina & 0,7 & 0,9 & $0,5-1,5 \mathrm{mg} / \mathrm{dL}$ \\
Fosfatase Alcalina (FA) & 237 & Soro discretamente ictérico & $0-156 \mathrm{U} / \mathrm{L}$ \\
\hline Observaçós do hem
\end{tabular}

Observações do hemograma: D1- Eritrócitos com intensa policromatofilia e moderada anisocitose. Metarrubricito (8\%). Foram visualizadas inclusões leucocitárias compatíveis com mórula de Erlichia canis. D2- Eritrócitos com moderada anisocitose e policromasia. Presença de eritrócitos com hipocromia (++); metarrubricitos (20\%) e neutrófilos hipersegmentados (+). Plasma discretamente ictérico. 
Após constatar a inexistência de sangramento abdominal, iniciou-se o fechamento da cavidade. A musculatura da parede abdominal foi suturada com fio de sutura sintético absorvível 2-0 ácido poliglicólico - Vicryl ${ }^{\circledR}$ utilizando sutura descontínua de Sultan (em X). Em seguida, foi realizada a redução do espaço morto com o mesmo fio e padrão de sutura contínua simples. A pele foi suturada com fio de sutura sintético não absorvível 2-0 Nylon ${ }^{\circledR}$ e padrão de sutura descontínua de Wolff (U deitado).

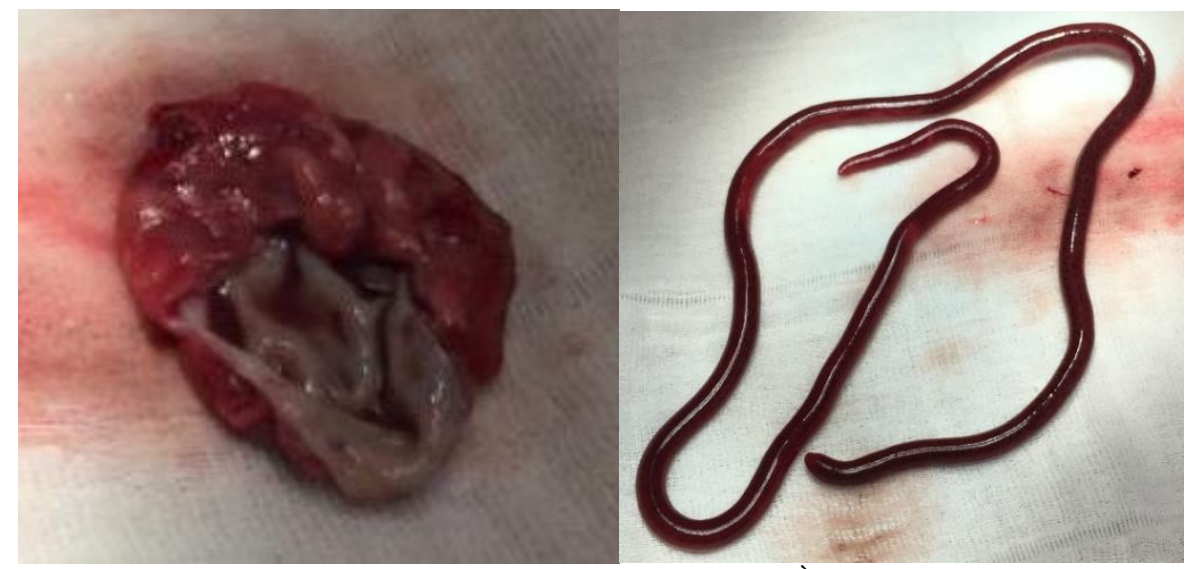

Figura 2. Aspecto do rim acometido no pós-cirúrgico imediato. À esquerda pode ser observada a cápsula renal aberta, com severa perda dos aspectos da anatomia interna do órgão. À direita se evidencia o parasito de aspecto cilíndrico, coloração avermelhada e extenso comprimento.

No pós-operatório, foi realizado novo hemograma (Tabela 2) que evidenciou leucocitose. Além disso, o paciente apresentou os seguintes sinais clínicos: fraqueza, apatia, colúria e icterícia que foi observada nas mucosas oculares, oral e na pele. Devido a outras complicações no quadro clínico do paciente, o animal morreu após alguns dias da realização da nefrectomia.

Tabela 2. Resultados de exames laboratoriais (hemograma) do paciente após a realização da nefrectomia. Valores de referência: Jain (1993) e Kaneko et al. (2008).

\begin{tabular}{lcc}
\hline Parâmetro & Resultado & Referência \\
\hline Hematócrito $(\mathrm{Ht})$ & 21 & $37-55 \%$ \\
Hemácias & 2,68 & $5,5-8,5 \mathrm{milhões} / \mathrm{mm}^{3}$ \\
Hemoglobina $(\mathrm{Hb})$ & 6,4 & $12-18 \mathrm{~g} / \mathrm{dL}$ \\
Volume corpuscular médio & 78,4 & $60-77 \mathrm{fL}$ \\
Concentração de hemoglobina corpuscular média & 30,5 & $32-36 \%$ \\
Leucometria global & 38.100 & $6.000-17.000 / \mathrm{mm}^{3}$ \\
Metamielócitos & 0 & $0 / \mathrm{mm}^{3}$ \\
Bastões & 0 & $0-510 / \mathrm{mm}^{3}$ \\
Neutrófilos segmentados & 30.480 & $3.000-11.500 / \mathrm{mm}^{3}$ \\
Linfócitos & 1.905 & $1.000-4.800 / \mathrm{mm}^{3}$ \\
Monócitos & 5.334 & $150-1.350 / \mathrm{mm}^{3}$ \\
Eosinófilos & 381 & $100-1.250 / \mathrm{mm}^{3}$ \\
Basófilos & 0 & $0-170 / \mathrm{mm}^{3}$ \\
PPT & 5,8 & $54-71 \mathrm{~g} / \mathrm{dL}^{3}$ \\
Plaquetas & 157.000 & $144.000-510.000 / \mathrm{mL}^{3}$ \\
Observações do hemograma:Eritrócitos anisocitose $(++)$, policromasia $(++)$. Metarrubricitos $(5 \%)$. Icterícia $(+)$.
\end{tabular}

\section{Discussão}

Nesse relato, o diagnóstico de dioctofimose foi por meio do exame ultrassonográfico que é uma forma a qual permite a visualização dos parasitos no rim e na cavidade abdominal (Monteiro, 2011; Rappeti et al., 2016; Silveira et al., 2015). Na ultrassonografia observou-se o rim esquerdo em estado normal enquanto no rim direito havia indícios do parasitismo por $D$. renale, o que corrobora com as descrições encontradas na literatura, onde normalmente apenas um rim é afetado (Taylor et al., 2017) e que o rim direito é o mais comum devido à sua proximidade com o duodeno (Mesquita et al., 2014). 
O cão descrito foi infectado pelo parasito por meio da ingestão do anelídeo junto com a água ou pela ingestão da carne dos hospedeiros paratênicos infectados, como rãs ou peixes, que são os meios que os animais podem se infectar com o parasito (Pedrassani, 2009), e por ser um animal de rua, possivelmente entrou em contato com esses hospedeiros intermediários em algum ambiente contaminado como restos de lixo ou margens de rios.

Os sinais clínicos apresentados pelo animal, como fraqueza e apatia, são alguns dos mais relatados em animais com esse diagnóstico (Lemos et al., 2010), apesar de que a maioria dos casos podem ser assintomáticos (Taylor et al., 2017).

No caso descrito, o parasito apresentava $70 \mathrm{~cm}$ de comprimento, correlacionando-se com as descrições encontradas na literatura, onde as fêmeas podem chegar até 1 metro de comprimento, o que as diferencia dos machos que medem em torno de $40 \mathrm{~cm}$ e possuem uma bolsa copuladora, semelhantes à um sino, a qual não foi encontrada no parasito do caso relatado (Monteiro, 2011).

O parênquima foi destruído por completo, restando apenas a cápsula renal. Eventualmente isso é o que ocorre até que permaneça apenas uma túnica que contenha o verme e exsudatos (Ferreira et al., 2010). Conforme já descrito, não existe tratamento clínico, apenas a realização cirúrgica com a remoção do parasito.

\section{Conclusão}

A ultrassonografia é um método essencial para o diagnóstico da dioctofimose. A demora no diagnóstico conduz ao prognóstico ruim, conforme observado no desfecho deste caso.

\section{Referências}

Birchard, S. J., \& Sherding, R. G. (2008). Manual Saunders: clínica de pequenos animais. In Ed. Roca (Vol. 3).

Colpo, C. B., Silva, A. S. S., Monteiro, S. G., Stainki, D. R., Camargo, D. G., \& Colpo, E. T. B. (2007). Ocorrência de Dioctophyma renale em cães no município de Uruguaiana-RS. Revista Da FZVA, 14(2), 175-180.

Ferreira, V. L., Medeiros, F. P., July, J. R., \& Raso, T. F. (2010). Dioctophyma renale in a dog: Clinical diagnosis and surgical treatment. Veterinary Parasitology, 168(1-2), 151-155. https://doi.org/10.1016/j.vetpar.2009.10.013

Jain, N. C. (1993). Essentials of Veterinary Hematology (1st ed.). Wiley-Blackwell.

Kaneko, J. J., Harvey, J. W., \& Bruss, M. L. (2008). Clinical biochemistry of domestic animals (6th Ed. (ed.); Vol. 1). Academic press.

Katafigiotis, I., Fragkiadis, E., Pournaras, C., Nonni, A., \& Stravodimos, K. G. (2013). A rare case of a 39year old male with a parasite called Dioctophyma renale mimicking renal cancer at the computed tomography of the right kidney. A case report. Parasitology International, 62(5), 459-460. https://doi.org/10.1016/j.parint.2013.06.007

Lemos, L. S., Santos, A. S. O., Rodrigues, A. B. F., Goulart, M. L. V. S., Almeida, L. G., \& Silveira, L. S. (2010). Extra-renal lesion caused by Dioctophyma renale eggs in an erratic cycle in a dog. Int. $j$. Morphol, 28(4), 1031-1034.

Mesquita, L. R., Rahal, S. C., Faria, L. G., Takahira, R. K., Rocha, N. S., Mamprim, M. J., \& Oliveira, H. S. (2014). Pre- and post-operative evaluations of eight dogs following right nephrectomy due to Dioctophyma renale. Veterinary Quarterly, 34(3), 167-171. https://doi.org/10.1080/01652176.2014.924166

Monteiro, S. G. (2011). Parasitologia na medicina veterinária (Vol. 1). Roca.

Monteiro, S. G., Sallis, E. S. V., \& Stainki, D. R. (2002). Infecção natural por trinta e quatro helmintos da espécie Dioctophyma renale (Goeze, 1782) em um cão. Revista Da FZVA, 9(1), 9.

Nakagawa, T. L. D. R., Bracarense, A. P. F. R. L., Reis, A. C. F., Yamamura, M. H., \& Headley, S. A. (2007). Giant kidney worm (Dioctophyma renale) infections in dogs from Northern Paraná, Brazil. Veterinary Parasitology, 145(3-4), 366-370.

Pedrassani, D. (2009). Aspectos morfológicos, imunológicos e epidemiológicos do Dioctophyme renale 
em cães no distrito de São Cristóvão, Três Barras, Santa Catarina. Tese de Doutorado Em Medicina Veterinária Preventiva, Faculdade de Ciências Agrárias e Veterinárias, Universidade Estadual Paulista, Jaboticabal, SP. 118p.

Rappeti, J. C., Mascarenhas, C. S., Perera, S. C., Müller, G., Grecco, F. B., Silva, L. M. C., Sapin, C. F., Rausch, S. F., \& Cleff, M. B. (2016). Dioctophyme renale (Nematoda: Enoplida) in domestic dogs and cats in the extreme south of Brazil. Revista Brasileira de Parasitologia Veterinária, 26(1), 119121. https://doi.org/10.1590/s1984-29612016072

Ruiz, M. F., Zimmermann, R. N., Aguirre, F. O., Stassi, A. F., \& Forti, M. S. (2014). Dioctofimosis: presentación de un caso clínico. Veterinaria Argentina S.R.L.; Revista Veterinaria Argentina;, 31, 315.

Silveira, C. S., Diefenbach, A., Mistieri, M. L., Machado, I. R. L., \& Anjos, B. L. (2015). Dioctophyma renale em 28 cães: aspectos clinicopatológicos e ultrassonográficos. Pesquisa Veterinária Brasileira, 35(11), 899-905. https://doi.org/10.1590/S0100-736X2015001100005

Taylor, M. A., Coop, R. L., \& Wall, R. L. (2017). Parasitologia Veterinária. Guanabara Koogan.

Zardo, K. M., Santos, D. R., Babicsak, V. R., Belotta, A. F., Oliveira, H. S., Estanislau, C. A., Mamprim, M. J., \& Brandão, C. V. S. (2012). Aspecto ultrassonográfico da dioctofimose renal canina. Veterinária e Zootecnia, 19(1-S. 1), 57-60.

Recebido: 21 de abril, 2020 .

Aprovado: 27 de maio, 2020.

Disponível online: 8 de outubro, 2020.
Licenciamento: Este artigo é publicado na modalidade Acesso Aberto sob a licença Creative Commons Atribuição 4.0 (CC-BY 4.0), a qual permite uso irrestrito, distribuição, reprodução em qualquer meio, desde que o autor e a fonte sejam devidamente creditados. 